Service social

\title{
The Making of Masculinities - The New Men's Studies, sous la direction de Harry BROD, Boston, Allen \& Unwin, 1987, 346 pages.
}

\section{Jocelyn Lindsay}

Volume 37, numéro 1-2, 1988

Par-delà les barrières des sexes

URI : https://id.erudit.org/iderudit/706397ar

DOI : https://doi.org/10.7202/706397ar

Aller au sommaire du numéro

Éditeur(s)

École de service social de l'Université Laval

ISSN

1708-1734 (numérique)

Découvrir la revue

Citer ce compte rendu

Lindsay, J. (1988). Compte rendu de [The Making of Masculinities - The New Men's Studies, sous la direction de Harry BROD, Boston, Allen \& Unwin, 1987, 346 pages.] Service social, 37(1-2), 269-271. https://doi.org/10.7202/706397ar d'utilisation que vous pouvez consulter en ligne.

https://apropos.erudit.org/fr/usagers/politique-dutilisation/ 


\section{RECENSIONS}

The Making of Masculinities - The New Men's Studies, sous la direction de Harry BROD, Boston, Allen \& Unwin, 1987, 346 pages.

Les études sur la condition masculine (men's studies) sont relativement récentes comme champ constitué, mais elles ont néanmoins suffisamment maturé pour subir une certaine évaluation. Voilà le point de départ indiqué par Harry Brod, qui a dirigé l'élaboration de ce volume destiné à faire le point sur ces études dans différents champs scientifiques.

Même si elles semblaient porter sur la condition masculine, la tendance des études traditionnelles à parler de l'homme comme la norme humaine a exclus de considérer ce qui était spécifique aux hommes. Elles ont produit une distorsion dans notre compréhension de ce qui est générique à l'humanité et elles ont freiné l'étude de la masculinité comme expérience spécifique, qui se situe aussi dans des contextes sociaux, historiques et culturels.

Ce volume comprend quatorze textes d'auteurs différents regroupés en cinq parties. Pour chacune de celles-ci, nous allons résumer un seul texte compte tenu de l'abondance du matériel.

La première partie fait une fresque, à la fois historique et prospective, des études sur la condition masculine. Ainsi, dans le texte de Joseph Pleck, on voit que la théorie de l'identité masculine liée au rôle sexuel (theory of male sex-role identity) a été très importante en sciences sociales dans les cinquante dernières années. Dans cette perspective, les individus vont acquérir une maturité en acquérant une identité homme ou femme, en manifestant les traits, attitudes, intérêts appropriés à leur sexe, qui valident leur entité biologique. Entre les années 1936 et 1945, on a assisté à un développement explicite, notamment par la psychométrie, de la configuration normative des caractéristiques des membres de chaque sexe. Dans cette période, les conceptions ayant comme pôles la masculinité et la féminité, s'appliquaient également aux deux sexes; mais entre 1945 et 1970, on a davantage insisté sur une conception de la masculinité, autant à cause des bouleversements sociaux que de nouveaux cadres théoriques comme ceux de l'identification parentale, de l'hypermasculinité comme défense contre l'identification féminine, et de l'absence parentale comme cause de la déviation et de la délinquance. À compter de 1970, on a noté un déclin du paradigme de l'identité liée au rôle sexuel. De plus en plus de recherches montrent que la conception bipolaire masculinité/féminité ne prédisait pas l'ajustement psychologique. Eu égard au sexe, on distingua de plus en plus entre l'identité (la conscience de l'individu d'être homme ou femme, et la satisfaction qu'il en retire) et le rôle (jusqu'à quel point l'individu possède les traits, attitudes et intérêts qu'on attend de lui dans telle culture). Les recherches sur l'androgynie ont aussi légitimé la présence chez une personne de traits associés à l'autre sexe. 
Enfin, le courant féministe a plus massivement remis en question les modèles de rôles traditionnels. Selon Pleck, la théorie de l'identité liée au rôle sexuel n'est plus dominante : elle fait plutôt partie de l'histoire.

La seconde partie inclut différentes thèses à caractère historique. Pour un, Michael Kimmel démontre des répétitions cycliques dans les changements. II évoque des similitudes importantes à trois périodes dans lesquelles les genres masculin et féminin ont été remis en question : l'Angleterre de 1700, les ÉtatsUnis du début du vingtième siècle, et l'époque contemporaine dans le même pays. Selon lui, les changements dans la masculinité se produisent en réaction à ceux de la féminité, en réponse à des renégociations de statut inter-groupes, et à des obligations provoquées par les bouleversements économiques et sociaux.

La troisième partie s'intéresse aux façons dont les hommes en arrivent à forger leur masculinité, dans le travail comme dans le jeu. À partir d'une analyse de cas, Richard Ochberg étudie comment les hommes se perdent eux-mêmes dans leur travail. Les hommes sentent à la fois un besoin d'intégrer et de séparer les sphères du privé et du travail : il en résulte une tension psychologique, et la perception de leur performance dans différents rôles simultanés mène à l'anxiété et au stress. Les difficultés de s'appuyer sur des modèles d'une génération à l'autre, et la forte présence de la compétition influencent aussi leur cheminement de carrière.

La quatrième partie se réfère à différents cadres scientifiques (psychologie sociale, anthropologie, biosociologie) pour expliquer l'attachement que l'homme établit. Par exemple, Perry Treadwell examine les études selon lesquelles des quantités différentes de testostérone et d'œestrogène modifient, chez l'individu, l'expression de la masculinité ; ces hormones influencent la structuration du cerveau avant la naissance et, plus tard, l'expression d'agressivité. L'auteur n'avance pas ici un déterminisme biologique, mais pense plutôt à l'émergence d'une grande diversité de réponses physiologiques conditionnées par les environnements sociaux ; ainsi, on pourrait sélectionner, pour les sports ou l'entraînement militaire, des sujets plus à risque de répondre d'une façon plus stéréotypée aux environnements sociaux.

Dans la dernière partie, on fait des analyses de différents travaux littéraires américains et de leurs auteurs: le prisme d'une lecture masculiniste permet de révéler des significations et des masques de la masculinité, à la fois importants et subtils. Ainsi, James Riemer soutient qu'un tel exercice permet de faire une relecture d'une bonne portion de la littérature américaine en tant que documentation sociale qui reflète des variations dans les idéaux et dans les rôles masculins, en tenant aussi compte des variables sociales, économiques, raciales et ethniques.

En résumé, il faut reconnaître beaucoup d'audace à Harry Brod pour affirmer que les études sur la condition masculine ont atteint un degré de développement qui permet une articulation interne et une analyse de son évolution et de ses possibilités. De même, la construction d'un ouvrage sur ce sujet dans une optique pluridisciplinaire était à la fois difficile et inévitable. Il ne faut évidemment pas prétendre ici à une conception définitive de ce champ; 
mais tout au moins pouvons-nous croire en un volume représentant une étape importante dans le renouvellement des études sur la condition masculine; celles-ci apporteront une contribution, d'une part distincte des travaux féministes des deux dernières décades mais, d'autre part, complémentaires à ceux-ci.

Jocelyn LINDSAY

École de service social,

Université Laval.

The Woman Client - Providing Human Services in a Changing World, par D.S. Burden et N. Gottueb (éds.), New York, Tavistock Publication, 1987.

Ce volume se situe dans la continuité de l'ouvrage de E. Norman et A. Mancuso (Women's Issues and Social Work Practice, Itasca (III.), Peacock, 1980), première production traitant spécifiquement de la femme et du service social. Le texte vise à fournir un ancrage pour l'amélioration de l'enseignement, de la recherche et de l'intervention concernant les femmes. Il est proposé de tenir compte des changements survenus dans les aspects privés et politiques de leur vie. Les résultats de recherches empiriques portant sur les problématiques vécues par elles doivent également inspirer l'enseignement, la recherche et la pratique. D'autres disciplines intervenant auprès des femmes peuvent aussi faire grand usage de ce livre.

Il faut mentionner au départ que le volume est écrit par un groupe d'auteures. L'abondance des thèmes abordés est compensée par la clarté de la division en quatre parties (éléments génériques, intervention, problèmes et services, types de population), par la précision de la présentation de chacune des parties et par la rigueur de la synthèse qu'offre chacun des chapitres. Sans être toujours identiques, les rubriques utilisées permettent rapidement de se situer. Incidemment, la cueillette de toutes les recommandations pour l'enseignement pourrait constituer un curriculum féministe. Les références bibliographiques complétant chacun des chapitres sont très pertinentes et généralement récentes.

En introduction, on trouve une définition du sexisme :

"Le sexisme est un système non fonctionnel par lequel on utilise des stéréotypes liés au sexe; il tend à déprécier les femmes et à faire de la discrimination contre elles. Ce n'est pas quelque chose qui est fait aux femmes par les hommes, mais plutôt un système affectant à la fois hommes et femmes, le système d'éducation et la littérature. Le sexisme fait partie d'un système culturel dans lequel on perçoit les hommes comme ayant plus de valeur que les femmes." (P.2)

Le service social est interpellé quant au sexisme. Les auteures examinent les effets de ce phénomène sur la vie des clientes et des intervenantes, le rôle du sexisme dans les interventions, sa présence dans les organisations, le sexisme en relation avec la santé mentale des hommes et des femmes et le sexisme comme système politique. 